\title{
Der Selbstkonzept-Zug und das Schlangen-und- Leiter-Spiel in der psychomotorisch orientierten Selbstkonzeptförderung
}

Aufbauend auf dem Beitrag »Selbstkonzeptförderung in der Psychomotorik bei Kindern mit Duchenne Muskeldystrophie« in diesem Heft, wird in einem Spieletipp zunächst eine Möglichkeit erläutert, über eine symbolische Konstruktion psychomotorisch orientierte Selbstkonzeptförderung über mehrere Stunden zu umrahmen. Anschließend wird beispielhaft ein Spiel detaillierter beschrieben.

In der ersten Stunde wird mit den Kindern gemeinsam aus unterschiedlichen Materialien (z.B. Schwämme, kleine Schaumstoffbausteine, Korken, Wäscheklammern usw.) ein Zug gebaut. Jeder Waggon des Zuges soll eine Dimension des Selbstkonzepts repräsentieren (akademisches, soziales, emotionales und physisches), wobei die anführende Lok das allgemeine Selbstkonzept darstellt. Dieser Zug wird zu Beginn jeder Stunde angesprochen und daran erinnert, in welchem Waggon sich die Kinder heute befinden. Besprochen wird, welche Fähigkeiten ein Mensch haben kann und wo er diese zeigt oder benötigt (Schule, Freizeit, Freunde, Familie etc.). Die Metapher des Zuges ist aus dem Selbstkonzeptförderprogramm von Hugo (2005) in leicht veränderter Form übernommen.

Ein Spiel zum sozialen Selbstkonzept ist das Schlangen-und-Leiter-

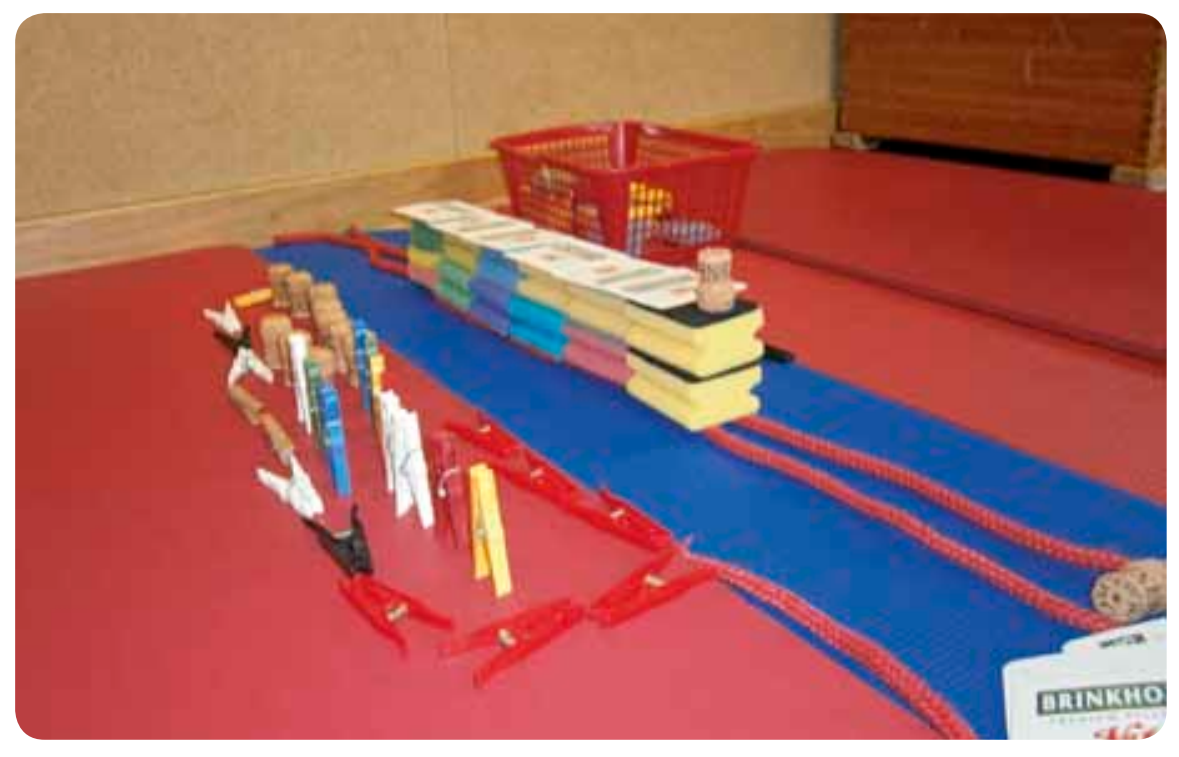

Abb.1: Selbstkonzept-Zug

Spiel. In dieser Stunde soll eine Kleingruppe von Kindern (ca. zwei bis vier) aus unterschiedlichen Materialien (Teppichfliesen, Seile, Papprohre) ein Spielfeld bauen. Dies geschieht in Anlehnung an das Schlangen-und-Leiter-Spiel (s. Abb.2). Das Schlangenund-Leiter-Spiel besteht aus beliebig vielen Feldern (eine Mindestzahl von ca. 20 Feldern ist empfehlenswert). Diese werden in Form von Teppichfliesen als Weg im Raum verteilt, wobei es eine Start- und eine Zielfliese gibt. Die Start- und Zielfliesen können ggf. besonders gekennzeichnet werden. Einzelne Teppichfliesen werden mit Seilen verbunden, andere mit Papprohren. Jeder Spieler hat eine Spielfigur (z.B.
Hütchen, Kegel, selbst gebastelte Figur) oder stellt selbst die Spielfigur dar. Während des Spiels würfeln die Spieler reihum und gehen dementsprechend auf den Spielfeldern vor.

Erreicht ein Spieler ein Feld, auf dem ein Seil hoch zu einer anderen Teppichfliese führt, darf er auf diese vorrücken. Landet ein Spieler jedoch auf einem Spielfeld, auf dem ein Papprohr nach unten zu einem vorherigen Feld führt, muss er auf dieses zurückgehen. Das Spiel kann je nach Altersgruppe so gestaltet werden, dass die Spieler nach einem Wurf gemeinsam entscheiden, welcher Spieler vorzieht, damit niemand auf eine Fliese mit einem Papprohr gelangt bzw. wenn 
möglich ein Spieler auf ein Feld mit einem Seil gehen kann. Ziel ist es, dass jeder Mitspieler die Zielfliese erreicht. Mit diesem Spiel wird den Kindern verdeutlicht, dass es sowohl positive als auch negative Beziehungen gibt (Hugo 2005, 24). Beim Spielen des Spiels mit großen Softwürfeln gibt es die Möglichkeit, diese Qualitäten von Beziehungen sowohl körperlich, spielerisch zu erfahren als auch verbal zu thematisieren.

\section{(10国) Literatur}

Hugo, L. (2005): The development and evaluation of a self-concept enrichment programme for children aged 7-9 years. Mini-dissertation, NordWest University, Potchefstroom

\section{Marianne Irmler}

DOI 10.2378 / motorik2015.arto6d

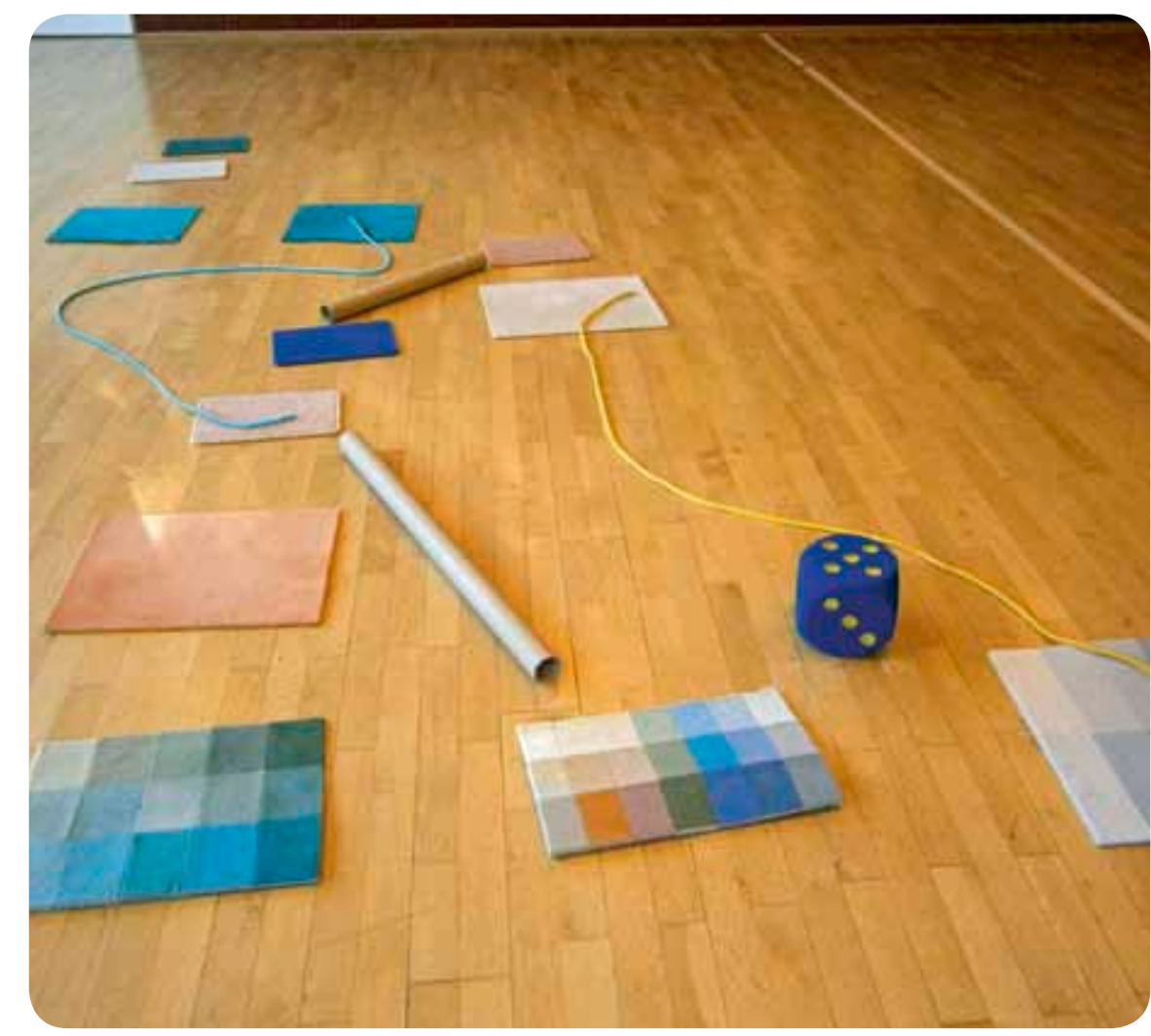

Abb. 2: Schlangen-und-Leiter-Spiel

\section{Praxisbeispiele zur Förderung des Selbstkonzepts in und durch Bewegung}

Die Entwicklung eines positiven Selbstkonzeptes ist ein zentrales Anliegen in der kindzentrierten psychomotorischen Entwicklungsförderung nach Zimmer (2012). Körperliche Erfahrungen sind für Kinder wichtig, um Kenntnisse über sich und die eigenen Fähigkeiten zu gewinnen. Psychomotorisch begleitete Bewegungsspiele scheinen somit äußerst geeignet, um den Aufbau eines positiven Selbstkonzeptes zu unterstützen. An dieser Stelle werden zwei Spiele exemplarisch vorgestellt, die Bestandteil der psychomotorischen Förderpraxis im Rahmen einer Pilotstudie zu Veränderungen im Selbstkonzept waren (Ruploh et al.
2013). Die Spielideen sind an anderer Stelle bereits veröffentlicht (Zimmer 2012, $218 \mathrm{ff}$ ), werden hier jedoch mit konkreten spezifischen Vorteilen der beiden Spiele im Hinblick auf das Ziel der Stärkung eines positiven Selbstkonzeptes vertiefend beschrieben. Ergänzend werden Variationsmöglichkeiten aufgezeigt, die sich gemeinsam im Prozess mit den Kindern entwickeln können.

\section{Schlangenfangen}

Bei dem Spiel "Schlangenfangen« bekommt jedes Kind ein Seil, das es an einem Ende festhält und während des
Laufens durch Hin- und Her-Bewegen in eine Schlange verwandelt, die sich dadurch schlängelnd über den Boden fortbewegt. Wichtig ist dabei der Hinweis, dass nur »Wüstenschlangen « entstehen, damit die Kinder das Seil lediglich auf dem Boden bewegen und gefährliche, peitschenartige Bewegungen des Seils in der Luft ausbleiben. Die erwachsene Begleitperson ist der Schlangenfänger und versucht durch das Treten auf eine Schlange, diese zu fangen. Das Fangen muss zunächst keine Auswirkung auf den weiteren Verlauf des Spiels haben, solange der Erwachsene durch sein dosiertes Fangen die Spielmotivation aller Kinder indivi- 